\title{
MST1 inhibits the progression of breast cancer by regulating the Hippo signaling pathway and may serve as a prognostic biomarker
}

\author{
XIANG JIN, LIHUA ZHU, SHENG XIAO, ZHUHONG CUI, JING TANG, JIANGYONG YU and MINGJUN XIE
}

Department of Breast Surgery, The First People's Hospital of Yibin, Yibin, Sichuan 644000, P.R. China

Received July 28, 2020; Accepted February 18, 2021

DOI: $10.3892 / \mathrm{mmr} .2021 .12022$

\begin{abstract}
Breast cancer (BCa) is the most common malignancy threatening the health of women worldwide, and the incidence rate has significantly increased in the last 10 years. Mammalian STE20-like protein kinase 1 (MST1) is involved in the development of various types of malignant tumor. The present study aimed to investigate the role of MST1 in $\mathrm{BCa}$ and its potential involvement in the poor prognosis of patients with BCa. Reverse transcription-quantitative PCR and immunohistochemistry were used to analyze the expression levels of MST1 in BCa, and the clinicopathological characteristics and prognosis of patients with $\mathrm{BCa}$ were further analyzed by statistical analysis. MST1 was overexpressed in BCa cell lines (MCF-7, MDA-MB-231 and SKBR3). Cell Counting Kit-8, 5-ethynyl-2'-deoxyuridine and flow cytometry assays were used to analyze cell proliferation and apoptosis, respectively, and a wound healing assay was used to analyze cell migration. The results of the present study revealed that the downregulated expression levels of MST1 in BCa were closely associated with the poor prognosis of patients, and MST1 may be an independent risk factor for BCa. The overexpression of MST1 significantly inhibited the proliferation and migration, and promoted the apoptosis of BCa cells. In addition, the overexpression of MST1 significantly activated the Hippo signaling pathway. Treatment with XMU-MP-1 downregulated the expression levels of MST1 and partially reversed the inhibitory effects of MST1 on proliferation, migration and apoptosis-related proteins, and inhibited the Hippo signaling pathway. In conclusion, the results of the present study suggested that MST1 expression levels may be downregulated in $\mathrm{BCa}$ and closely associated with tumor size and clinical stage, as well as the poor prognosis of affected
\end{abstract}

Correspondence to: Professor Mingjun Xie, Department of Breast Surgery, The First People's Hospital of Yibin, 65 Wenxing Street, Cuiping, Yibin, Sichuan 644000, P.R. China

E-mail: xiemingjun886@126.com

Key words: mammalian STE20-like protein kinase 1, breast cancer, Hippo signaling, poor prognosis patients. Furthermore, MST1 may inhibit the progression of BCa by targeting the Hippo signaling pathway.

\section{Introduction}

Breast cancer $(\mathrm{BCa})$ is one of the most common types of malignant tumor in women $(1,2)$. There were 2.26 million new cases of BCa worldwide in 2019, accounting for $24.5 \%$ of all female-related cancers worldwide. BCa also accounted for 680,000 deaths in 2019, ranking as the fifth most common cause of cancer-related mortality worldwide, and the incidence rate of $\mathrm{BCa}$ increases with age (2). Hereditary factors, infertility, an unhealthy lifestyle and excess mental stress are common risk factors for $\mathrm{BCa}$ (3). The global incidence rate of $\mathrm{BCa}$ is $24.2 \%$, ranking first among cancers affecting women (4). In China, 300,000 women are diagnosed with $\mathrm{BCa}$ each year. Following the development and implementation of novel treatment strategies, the mortality rate of $\mathrm{BCa}$ has gradually decreased $(4,5)$. However, in vast rural areas of China, the decreasing trend of BCa mortality is not significant, and still accounts for 120,000 deaths each year (6). Symptoms of early $\mathrm{BCa}$ are not obvious, whereas distant metastasis and multiple organ disease often occur in advanced late-stage disease, which pose a significant threat to the lives of affected patients (7). Therefore, the molecular mechanisms underlying the occurrence and development of $\mathrm{BCa}$ must be identified to provide novel treatment options for $\mathrm{BCa}$.

Mammalian STE20-like protein kinase 1 (MST1) is a class II GC kinase belonging to the sterile (STE)-20 protein kinase family; it comprises 487 amino acid residues and has a molecular weight of $59 \mathrm{kDa}(8)$. MST1 is widely expressed in most cells of the human body and is a core component of the Hippo signaling pathway, which serves an important role in cell differentiation, adhesion, migration, apoptosis and other physiological activities through the phosphorylation, dimerization and nuclear localization of target proteins $(9,10)$. MST1 was reported to promote the differentiation of tumor cells and to regulate trophoblast differentiation and placental morphogenesis (11). Moreover, MST1 was previously reported to serve regulatory roles in cell adhesion, endocytosis and migration speed by modulating C-C motif chemokine receptor 7 (CCR7). Downregulated expression levels of MST1 also inhibited the phosphorylation of CCR7 in mature dendritic cells and reduced the migration speed of cells by downregulating the 
expression of curl protein, myosin 1 light chain and myosin 1 light chain phosphatase (12). Another previous study found that MST1 promoted the apoptosis of cardiomyocytes and islet cells (13). In diabetes, MST1 induced apoptotic cell death by upregulating the expression levels of the BCL2-like 11 protein in $\beta$ cells, and disrupting normal cardiac function (14).

The Hippo signaling pathway comprises a group of conserved kinases and is a key signaling pathway regulating cell proliferation (15). In mammals, the membrane protein receptor upstream of the Hippo signaling pathway senses the growth inhibition signal of the extracellular environment and then acts on downstream effectors, YY1-associated protein 1 (YAP) and tafazzin (TAZ) through a series of kinase-phosphorylation reactions (16). In the Hippo signaling pathway, MST1 binds to and activates the phosphorylation of large tumor suppressor kinase (LATS)1/2. YAP is the main downstream effector of this signaling pathway, and it is directly phosphorylated by LATS1/2, which subsequently inhibits the expression of YAP/TAZ. YAP can bind to the 14-3-3 protein in the phosphorylated state, translocate from the nucleus to the cytoplasm, and lose the transcriptional coactivator activity (17). Therefore, MST1 controls LATS1/2 and YAP activity by regulating YAP subcellular localization and protein stability. Long-term activation of YAP, such as its transgenic expression in the mouse liver, was found to lead to cell transformation and tumor development, indicating a role for the Hippo signaling pathway in tumorigenesis and development (18). YAP was found to upregulate zinc-finger E-box-binding homeobox 1 expression and promote epithelial-mesenchymal transition (EMT), a key step in tumor metastasis (19). In addition, YAP-induced genome instability, and its homologous protein TAZ was necessary to maintain BCa stem cell self-renewal and tumorigenesis (20). It was previously reported that YAP/TAZ activity was also associated with drug resistance and tumor recurrence, as $\mathrm{BCa}$ cells with high YAP/TAZ activity were resistant to paclitaxel, 5-fluorouracil and Adriamycin (21). Tamoxifen is an important drug commonly used to treat estrogen receptor (ER)-positive $\mathrm{BCa}$; however, some ER-positive BCas are not sensitive to tamoxifen (21). Tamoxifen was shown to activate YAP/TAZ by stimulating the membrane ER, G protein-coupled ER (22). Therefore, YAP may play an important role in the malignant development of BCa.

The present study aimed to investigate whether MST1 was an important biological indicator of the prognosis of $\mathrm{BCa}$ and to determine whether MST1 affected the biological functions of BCa through the Hippo signaling pathway. This research may provide novel insights and a basis for the further study of the molecular mechanisms of $\mathrm{BCa}$.

\section{Materials and methods}

Patient studies. A total of $75 \mathrm{BCa}$ and paired adjacent normal ( $>5 \mathrm{~cm}$ from the boundary of the tumor) tissues were obtained from female patients (age range, 27-79 years) with BCa who underwent surgical resection at The First People's Hospital of Yibin (Yibin, China) between October 2012 and May 2014. Patients were followed up to evaluate the survival rate of patients with $\mathrm{BCa}$ at 10 years post-surgery. Cases lost to follow-up or cases in which death occurred by causes other than $\mathrm{BCa}$ were censored in the survival analysis. The collected clinical $\mathrm{BCa}$ samples were stored at $-80^{\circ} \mathrm{C}$. The present study was approved by the Ethics Committee of The First People's Hospital of Yibin (approval no. 20121011). Written informed consent was obtained from all patients prior to participation in the study. The following exclusion criteria were used: i) Patients with incomplete pathological and clinical information; ii) patients with recurrent disease; iii) patients that had received preoperative radiation or chemotherapy, or any other treatment; iv) patients with other preoperative complications; v) patients with cognitive or mental disorders; and vi) pregnant women. The following inclusion criteria were used: i) $\mathrm{BCa}$ tissue samples were all pathologically and confirmed as $\mathrm{BCa}$.

Cell lines, culture and treatment. The human BCa cell lines, MCF-7, MDA-MB-231 and SKBR3, and the normal mammary epithelial cell line, MCF-10A, were obtained from the American Type Culture Collection. All cell lines were cultured in DMEM (Gibco; Thermo Fisher Scientific, Inc.) supplemented with 10\% FBS (Gibco; Thermo Fisher Scientific, Inc.), and maintained in a humidified incubator at $37^{\circ} \mathrm{C}$ with $5 \% \mathrm{CO}_{2}$.

XMU-MP-1 (cat. no. S8334; Selleck Chemicals), which is an inhibitor of MST1, was dissolved in DMSO and added to the medium at a final concentration of $0.1 \%$. Cell lines were cultured in the medium for $1 \mathrm{~h}$ at $37^{\circ} \mathrm{C}$.

Cell transfection. Lentivirus (LV) containing the MST1 sequence and negative control ( $\mathrm{NC}$; non-targeting) were purchased from Shanghai Gene Pharma Co., Ltd. Cells were seeded into six-well plates and cultured to $30-50 \%$ confluence. Subsequently, lentiviral transduction $(1 \mu \mathrm{g} / \mathrm{ml})$ was performed using Lipofectamine ${ }^{\circledR} 3000$ reagent (Invitrogen; Thermo Fisher Scientific, Inc.) according to the manufacturer's instructions. Following $48 \mathrm{~h}$ of transfection, reverse transcription-quantitative PCR (RT-qPCR) and western blotting were performed to analyze MST1 expression levels. Subsequent experiments were performed 24-72 $\mathrm{h}$ after transfection.

Immunohistochemistry staining. Briefly, tissues were fixed in $10 \%$ formalin for $12 \mathrm{~h}$ at room temperature and embedded in paraffin blocks for $8 \mathrm{~h}$ at room temperature. Tissue microarray (TMA) cores (1.5-mm diameter) were constructed from formalin-fixed paraffin-embedded BCa sections (4- $\mu \mathrm{m}$ thick). TMA slides were deparaffinized twice with xylene for $15 \mathrm{~min}$ each at room temperature and rehydrated three times in a descending series of ethanol solutions (100, 100, 95 and 80\%). Antigen retrieval was performed with citric acid buffer $(10 \mathrm{mM}$; $\mathrm{pH}$ 6.0) in a microwave $(800 \mathrm{~W})$ for $15 \mathrm{~min}$, and samples were then cooled at room temperature for $30 \mathrm{~min}$. All slides were incubated with 5\% goat serum (OriGene Technologies, Inc.) for $15 \mathrm{~min}$ at room temperature to block non-specific binding at room temperature, followed by an incubation with a rabbit monoclonal anti-MST1 antibody (1:200; cat. no. ab231138; Abcam) at $4^{\circ} \mathrm{C}$ overnight. Following the primary antibody incubation, slides were incubated with an anti-rabbit secondary IgG antibody (1:100; cat. no. SAP-9100; OriGene Technologies, Inc.) at $37^{\circ} \mathrm{C}$ for $60 \mathrm{~min}$. The membranes were then washed with PBS and stained with 3,3'-diaminobenzidine (cat. no. K5007; Dako; Agilent Technologies, Inc.) for $30 \mathrm{sec}$ at room temperature. Slides were visualized using a light microscope (magnification, x100; Zeiss AG). 
MST1 immunostaining results were evaluated by two blinded pathologists. MST1 staining was scored according to the percentage of positive cells and staining intensity. The scoring parameters for staining intensity were as follows: 0 , negative; 1 , weak; 2, moderate; and 3, strong. The scoring parameters for the percentage of positive cells were as follows: Negative, $0-5 \%$; $1,6-25 \% ; 2,26-50 \% ; 3,51-75 \%$; and 4, 76-100\%. Sections with a total combined score of $<4$ were characterized as low MST1 expression, while sections with a score of $\geq 4$ were characterized as high MST1 expression.

$R T$ - $q P C R$. Total RNA was extracted from tissues and cell lines using TRIzol ${ }^{\circledR}$ reagent (Invitrogen; Thermo Fisher Scientific, Inc.). Total RNA was reverse transcribed into cDNA using a PrimeScript RT Reagent kit (Takara Bio, Inc.) at $35^{\circ} \mathrm{C}$ for $10 \mathrm{~min}, 40^{\circ} \mathrm{C}$ for $30 \mathrm{~min}$ and $76^{\circ} \mathrm{C}$ for $10 \mathrm{~min}$. qPCR was subsequently performed using SYBR Premix Ex Taq II (Takara Bio, Inc.) on an ABI Prism 7500 Real-Time PCR system (Applied Biosystems; Thermo Fisher Scientific, Inc.). The following primer sequences were used for the qPCR: MST1 forward, 5'-ATTCGGCTACGGAACAAG-3' and reverse, 5'-AAA ACGGGGTCCCTATTA-3'; and GAPDH forward, 5'-GGA GCGACATCCGTCCAAAAT-3' and reverse, 5'-GGCTGT TGTCAATCTTCTCATGG-3'. The following thermocycling conditions were used for the qPCR: Initial denaturation at $90^{\circ} \mathrm{C}$ for $2 \mathrm{~min}$; followed by 40 cycles at $93^{\circ} \mathrm{C}$ for $15 \mathrm{sec}, 60^{\circ} \mathrm{C}$ for $30 \mathrm{sec}$ and $72^{\circ} \mathrm{C}$ for $30 \mathrm{sec}$. mRNA expression levels of MST1 were quantified using the $2^{-\Delta \Delta \mathrm{Cq}}$ method (23).

Bioinformatics analysis. Gene Expression Profiling Interactive Analysis (http://gepia.cancer-pku.cn/index.html), Clinical Proteomic Tumor Analysis Consortium (CPTAC; https://proteomics.cancer.gov/programs/cptac) and The Cancer Genome Atlas (TCGA; www.cancer.gov/about-nci/organization/ccg/research/structural-genomics/tcga) public databases were used to analyze MST1 expression in BCa and the prognostic value of MST1.

Western blotting. Total protein was extracted using RIPA lysis buffer (Beyotime Institute of Biotechnology). Total protein was quantified using the Bradford protein assay (Bio-Rad Laboratories, Inc.) and $50 \mu \mathrm{g}$ protein/lane was separated via $10 \%$ SDS-PAGE. The separated proteins were subsequently transferred onto PVDF membranes and blocked with 5\% skim milk powder at room temperature for $1 \mathrm{~h}$. The membranes were then incubated with the following primary antibodies at $4^{\circ} \mathrm{C}$ overnight: Anti-MST1 (1:3,000; cat. no. ab231138; Abcam), anti-LATS1 (1:5,000; cat. no. ab70562; Abcam), anti-YAP (1:5,000; cat. no. ab205270; Abcam), anti-Bax (1:5,000; cat.no.ab32503; Abcam), anti-Bcl-2 (1:1,000; cat.no. ab182858; Abcam), anti-Ki-67 (1:1,000; cat. no. ab92742; Abcam) and anti- $\beta$-actin (1:5,000; cat. no. ab8226; Abcam). Following the primary antibody incubation, the membranes were incubated with secondary antibodies (1:5,000; cat. nos. ab6721 and ab6789; Abcam) at room temperature for $1 \mathrm{~h}$. Protein bands were visualized using enhanced chemiluminescence solution (EMD Millipore) and a ChemiDoc Imaging system (Bio-Rad Laboratories, Inc.). Protein expression was quantified using Quantity One version 4.6.5 software (Bio-Rad Laboratories, Inc.).
Cell Counting Kit-8 (CCK-8) assay. A total of 2x10 3 MCF-7 and SKBR3 cells/well were transfected with LV for $48 \mathrm{~h}$. Subsequently, $100 \mu \mathrm{l}$ cell suspension $\left(1 \times 10^{3}\right.$ cells/well) was plated into 96-well plates and incubated for 24, 48 and $72 \mathrm{~h}$ at $37^{\circ} \mathrm{C}$. Following each incubation, $10 \mu \mathrm{l} \mathrm{CCK}-8$ solution (Dojindo Molecular Technologies, Inc.) was added to each well and incubated for $1 \mathrm{~h}$ at $37^{\circ} \mathrm{C}$. The optical density of each well was measured at a wavelength of $450 \mathrm{~nm}$ using a microplate reader.

Wound healing assay. Briefly, $2 \mathrm{ml}$ cell suspension $\left(1 \times 10^{5}\right.$ cells/well) was plated into six-well plates and cultured in DMEM supplemented with $10 \% \mathrm{FBS}$ at $37^{\circ} \mathrm{C}$. After reaching $100 \%$ confluence, the cell monolayer was scratched with a $1-\mathrm{ml}$ pipette tip and then rinsed twice with PBS to remove nonadherent cells. Cells were subsequently cultured in serum-free DMEM at $37^{\circ} \mathrm{C}$. Following 0, 24 and $48 \mathrm{~h}$ of incubation, the distance between the two sides of the wound was visualized and photographed using a light microscope (magnification, x200). The cell migration rate (\%) was calculated using the following equation: [(Original gap distance-gap distance at each time point)/original gap distance] x100. ImageJ software (v2.1.4.7; National Institutes of Health) was used for analysis.

Flow cytometric analysis of cell apoptosis and the cell cycle. For cell apoptosis, $2 \mathrm{ml}$ cell suspension $\left(1 \times 10^{5}\right.$ cells/well) was plated into six-well plates. After reaching $100 \%$ confluence, cells were centrifuged at $200 \mathrm{x} \mathrm{g}$ for $5 \mathrm{~min}$ at room temperature. BCa cells were subsequently incubated with $5 \mu \mathrm{l}$ Annexin V-FITC and $5 \mu \mathrm{l}$ propidium iodide (PI) solution (Bioworld Technology, Inc.) for $15 \mathrm{~min}$ at $37^{\circ} \mathrm{C}$. Then, cells were suspended in $400 \mu \mathrm{l}$ binding buffer (Bioworld Technology, Inc.).

For analysis of cell cycle distribution, cells were cultured as described for cell apoptosis, centrifuged (200 x g; $5 \mathrm{~min}$; room temperature) and fixed in $75 \%$ ethanol at $-20^{\circ} \mathrm{C}$ overnight. The fixed cells were then washed with PBS and incubated with RNase A for $20 \mathrm{~min}$ at room temperature prior to being stained with PI in the dark for $30 \mathrm{~min}$ at $4^{\circ} \mathrm{C}$. Cell apoptosis and cell cycle distribution were analyzed using a FACSCalibur flow cytometer (BD Biosciences) and FACStation ${ }^{\mathrm{TM}} 6.1$ software (BD Biosciences).

5-Ethynyl-2'-deoxyuridine (EdU) incorporation assay. A total of $1 \times 10^{5}$ cells were plated into 96-well plates and cultured until they reached $30-50 \%$ confluence. Cells were then fixed with $4 \%$ paraformaldehyde at room temperature for $30 \mathrm{~min}$, and nuclei were permeabilized with $0.5 \%$ Triton X-100 solution for $30 \mathrm{~min}$ at room temperature. The cells were subsequently incubated with $50 \mu \mathrm{M}$ EdU, $1 \mathrm{X}$ ApolloR reaction cocktail $(100 \mu \mathrm{l})$ and $1 \mathrm{X}$ Hoechst $33342(100 \mu \mathrm{l})$ for $30 \mathrm{~min}$ at room temperature. Cell proliferation was measured by determining the mean number of the cells in three randomly selected fields of view from each sample using a fluorescence microscope (BioTek Instruments, Inc.; magnification, x100).

Statistical analysis. Statistical analysis was performed using SPSS version 22.0 software (SPSS, Inc.) and GraphPad Prism version 6.0 software (GraphPad Software, Inc.). Data are presented as the mean \pm SD. All experiments were repeated 
A

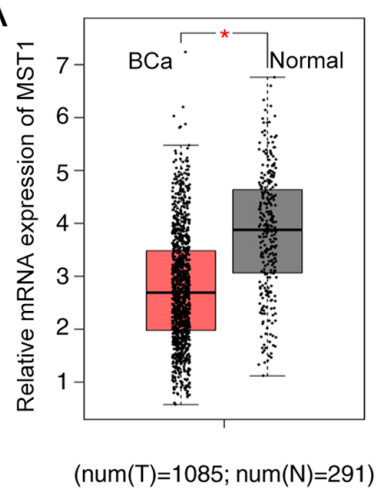

D

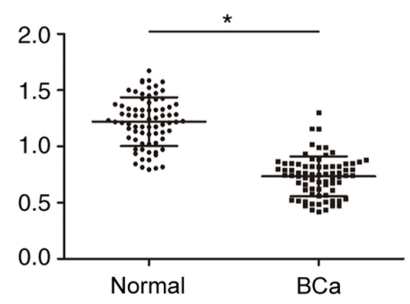

B

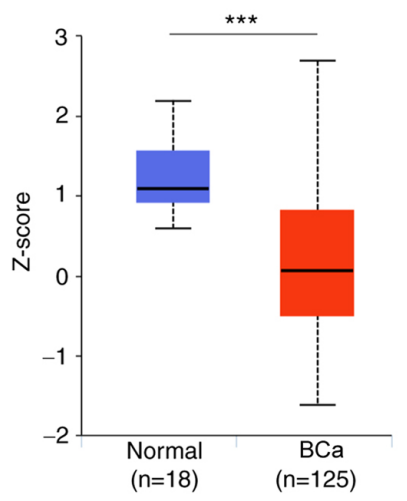

E

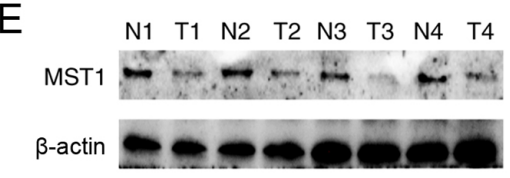

C
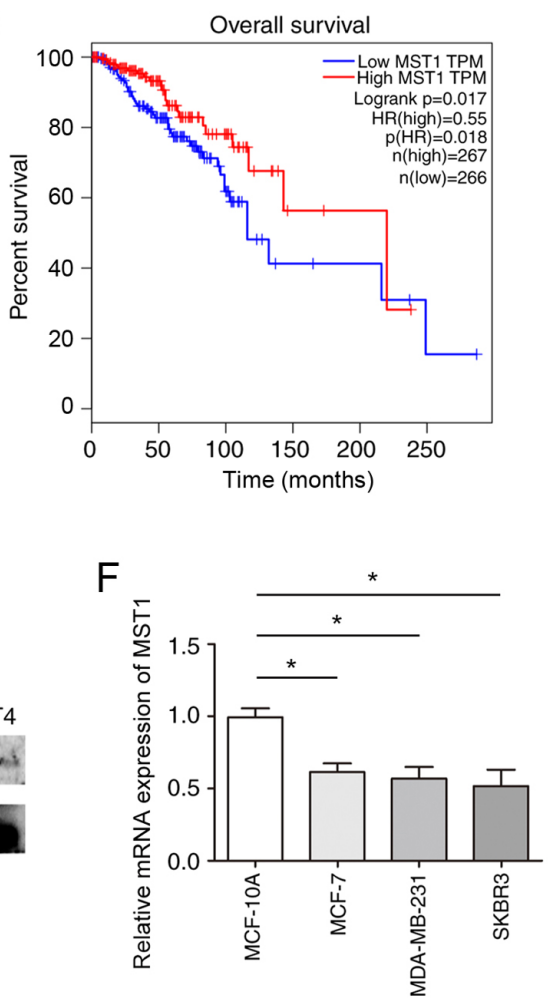

Figure 1. MST1 expression levels are downregulated in BCa tissues and cell lines. (A) Expression levels of MST1 in BCa and adjacent normal tissues were analyzed in a BCa dataset from TCGA (T=1,085; N=291). (B) Expression levels of MST1 in BCa and normal tissues obtained from the Clinical Proteomic Tumor Analysis Consortium public database. (C) Kaplan-Meier survival analysis and a log-rank test were performed to analyze the relationship between MST1 expression levels and the overall survival of patients using data from TCGA database (low MST1 expression, 266 patients; high MST1 expression, 267 patients). (D) RT-qPCR was used to analyze the mRNA expression levels of MST1 in BCa and adjacent normal tissues (n=75). (E) Western blotting was performed to determine the protein expression levels of MST1 in BCa and adjacent normal tissues. (F) RT-qPCR was performed to analyze the expression levels of MST1 in BCa cell lines. ${ }^{*} \mathrm{P}<0.05,{ }^{* * *} \mathrm{P}<0.001$. BCa, breast cancer; MST1, mammalian STE20-like protein kinase 1; N, normal; TCGA, The Cancer Genome Atlas; RT-qPCR, reverse transcription-quantitative PCR; T, tumor.

in triplicate. Statistical differences between two groups were analyzed using an unpaired or paired Student's t-test. Unpaired Student's t-tests were used to determine statistical in TCGA and CPTAC data. Associations between clinicopathological parameters and MST1 expression levels were analyzed using a $\chi^{2}$ test for small sample sizes. Kaplan-Meier survival analysis, followed by a log-rank test, and Cox univariate and multivariate regression analyses were used to analyze the prognostic significance of MST1. Statistical differences between multiple groups were determined using a one-way ANOVA followed by a Tukey's post hoc test. $\mathrm{P}<0.05$ was considered to indicate a statistically significant difference.

\section{Results}

MST1 expression levels are downregulated in BCa tissues and cell lines. The expression levels of MST1 were analyzed in BCa tissues obtained from TCGA database. MST1 expression levels were significantly downregulated in BCa tissues compared with the normal tissues (Fig. 1A). The protein expression levels of MST1 in BCa specimens from the Clinical Proteomic Tumor Analysis Consortium (CPTAC) (https://proteomics.cancer.gov/programs/cptac) public database were also analyzed, and the results showed that the expression levels of MST1 were downregulated in $\mathrm{BCa}$ tissues compared with normal tissues (Fig. 1B). In addition, patients with low expression levels of MST1 had a poor prognosis compared with patients with high MST1 expression levels (Fig. 1C). The expression levels of MST1 were subsequently analyzed in 75 tumor and non-tumoral tissues from patients with BCa from The First People's Hospital of Yibin to verify its expression in $\mathrm{BCa}$. The results demonstrated that MST1 mRNA expression levels were significantly downregulated in BCa tissues compared with normal tissues (Fig. 1D). The results of the western blotting analysis also showed that MST1 protein expression levels were notably downregulated in BCa tissues compared with normal tissues (Fig. 1E). The mRNA expression levels of MST1 were also downregulated in $\mathrm{BCa}$ cell lines compared with the normal mammary epithelial cell line, MCF-10A (Fig. 1F).

Downregulated expression levels of MST1 are associated with worse clinicopathological features in patients with BCa. According to the immunohistochemical staining, patients with BCa were divided into two groups: MST1 low expression and MST1 high expression (Fig. 2A). The results of the immunohistochemical staining for MST1 expression is presented in Fig. S1. The prognosis of patients with BCa with low MST1 expression was worse compared with patients with high expression (Fig. 2B). Furthermore, by analyzing the clinicopathological features of the two groups of patients, MST1 expression was found to be significantly associated with tumor 
Table I. Association between MST1 expression levels and clinicopathological features of patients with breast cancer.

\begin{tabular}{|c|c|c|c|c|}
\hline \multirow[b]{2}{*}{ Clinicopathological feature } & \multirow[b]{2}{*}{ Cases $(n=75)$} & \multicolumn{2}{|c|}{ MST1 expression } & \multirow[b]{2}{*}{ P-value } \\
\hline & & High $(n=26)$ & Low $(n=49)$ & \\
\hline Age, years & & & & 0.228 \\
\hline$<60$ & 39 & 16 & 23 & \\
\hline$\geq 60$ & 36 & 10 & 26 & \\
\hline Tumor size, $\mathrm{cm}$ & & & & $<0.001$ \\
\hline$\leq 2$ & 38 & 21 & 17 & \\
\hline$>2$ & 37 & 5 & 32 & \\
\hline Clinical stage & & & & 0.003 \\
\hline $\mathrm{I} / \mathrm{II}$ & 39 & 20 & 19 & \\
\hline III/IV & 36 & 6 & 30 & \\
\hline Lymph node metastasis & & & & 0.110 \\
\hline$\leq 3$ metastatic sites & 38 & 15 & 23 & \\
\hline$\geq 3$ metastatic sites & 37 & 11 & 26 & \\
\hline Estrogen receptor status & & & & 0.128 \\
\hline Positive & 40 & 17 & 23 & \\
\hline Negative & 35 & 9 & 26 & \\
\hline Progesterone receptor status & & & & 0.089 \\
\hline Positive & 48 & 20 & 28 & \\
\hline Negative & 27 & 6 & 21 & \\
\hline HER2 status & & & & 0.592 \\
\hline Positive & 43 & 16 & 27 & \\
\hline Negative & 32 & 10 & 22 & \\
\hline
\end{tabular}

MST1, mammalian STE20-like protein kinase 1.

A

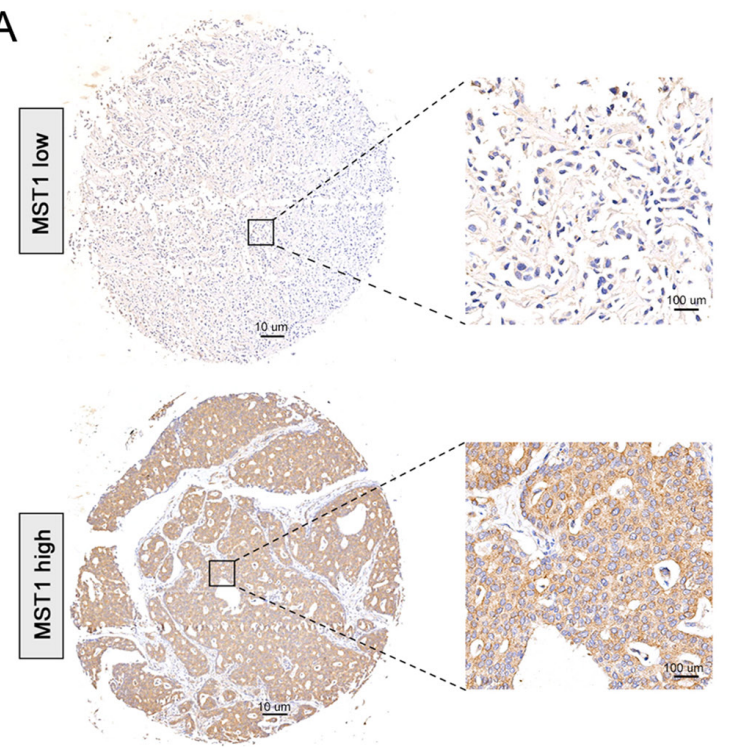

B

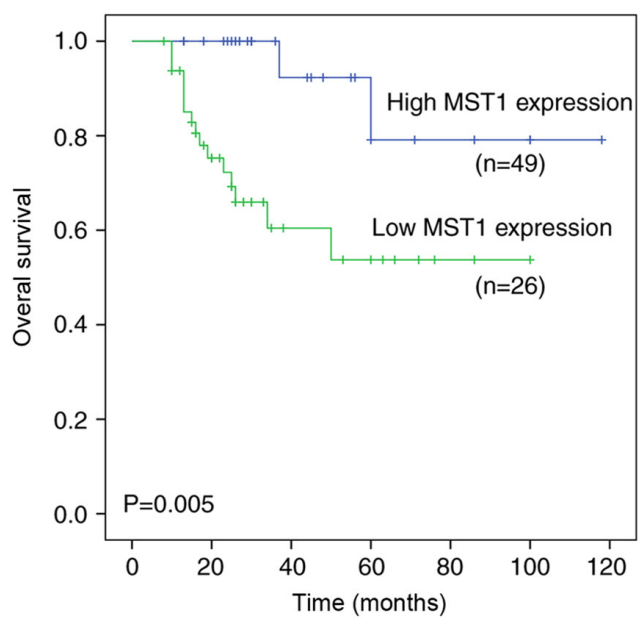

Figure 2. Effects of MST1 on the prognosis of patients with BCa. (A) Representative images of immunohistochemical staining showing high or low MST1 expression in BCa. Scale bars, 10- or 100- $\mu \mathrm{m}$. (B) Kaplan-Meier analysis followed by a log-rank test was used to determine the OS between patients with BCa with high and low MST1 expression. BCa, breast cancer; MST1, mammalian STE20-like protein kinase 1; OS, overall survival.

size $(\mathrm{P}<0.001)$ and clinical stage $(\mathrm{P}=0.003)($ Table $\mathrm{I})$. Through Cox univariate analysis, MST1 expression $(\mathrm{P}=0.005)$, tumor size (P 0.004) and clinical stage $(\mathrm{P}=0.019)$ were discovered to be significantly associated with overall survival (OS) 
Table II. Cox univariate and multivariate analyses of different prognostic variables influencing overall survival in patients with breast cancer.

\begin{tabular}{|c|c|c|c|c|c|}
\hline \multirow[b]{2}{*}{ Clinicopathological feature } & \multirow[b]{2}{*}{ Cases $(n=75)$} & \multicolumn{2}{|c|}{ Univariate analysis } & \multicolumn{2}{|c|}{ Multivariate analysis } \\
\hline & & $\mathrm{HR}(95 \% \mathrm{CI})$ & P-value & HR $(95 \%$ CI $)$ & P-value \\
\hline Age, years & & $0.630(0.448-1.064)$ & 0.736 & & \\
\hline$<60$ & 39 & & & & \\
\hline$\geq 60$ & 36 & & & & \\
\hline Tumor size, $\mathrm{cm}$ & & $1.397(0.655-3.845)$ & 0.004 & $1.048(0.596-3.674)$ & 0.010 \\
\hline$\leq 2$ & 38 & & & & \\
\hline$>2$ & 37 & & & & \\
\hline Clinical stage & & $1.554(1.078-3.994)$ & 0.019 & $1.484(0.980-3.481)$ & 0.013 \\
\hline $\mathrm{I} / \mathrm{II}$ & 39 & & & & \\
\hline III/IV & 36 & & & & \\
\hline Lymph node metastasis & & $1.073(0.761-2.663)$ & 0.166 & & \\
\hline$\leq 3$ metastatic sites & 38 & & & & \\
\hline$>3$ metastatic sites & 37 & & & & \\
\hline Estrogen receptor status & & $1.886(1.840-3.617)$ & 0.189 & & \\
\hline Positive & 40 & & & & \\
\hline Negative & 35 & & & & \\
\hline Progesterone receptor status & & $1.073(0.647-1.747)$ & 0.307 & & \\
\hline Positive & 48 & & & & \\
\hline Negative & 27 & & & & \\
\hline HER2 status & & $0.843(0.663-1.850)$ & 0.647 & & \\
\hline Positive & 43 & & & & \\
\hline Negative & 32 & & & & \\
\hline MST1 & & $1.308(0.537-2.478)$ & 0.005 & $1.186(0.634-2.705)$ & 0.009 \\
\hline Low & 49 & & & & \\
\hline High & 26 & & & & \\
\hline
\end{tabular}

MST1, mammalian STE20-like protein kinase 1; HR, hazard ratio; CI, confidence interval.

(Table II). Cox multivariate analysis revealed that OS was significantly associated with MST1 expression $(\mathrm{P}=0.009)$, tumor size $(\mathrm{P}=0.010)$ and clinical stage $(\mathrm{P}=0.013)$ (Table II), suggesting that MST1 expression may be an independent prognostic factor for OS in $\mathrm{BCa}$.

Overexpression of MST1 inhibits BCa cell proliferation, migration and apoptosis. MCF-7 cells had the highest expression of MST1 in the three tumor cell lines, while SKBR3 cells had the lowest expression of MST1 in the three tumor cell lines. Therefore, MCF-7 and SKBR3 cells were selected for use in subsequent experiments. LV-MST1 was used to successfully overexpress MST1 in BCa cells (Fig. 3A). The results of the CCK-8 assay revealed that MST1 overexpression significantly inhibited the proliferation of $\mathrm{BCa}$ cells compared with the NC group (Fig. 3B). The results of the EdU assay further verified that the overexpression of MST1 significantly inhibited cell proliferation compared with the NC group (Fig. 3C). The migration of BCa cells was analyzed using a wound healing assay, and the results revealed that cell migration was also significantly decreased following MST1 overexpression compared with the NC group at both 24 and 48 h (Fig. 3D). Furthermore, the effect of MST1 on the apoptosis of BCa cells was determined. Flow cytometric analysis revealed a significant increase in the apoptotic rate of both MCF-7 (8.5 vs. 1.4\%) and SKBR3 (9.8 vs. 3.6\%, ) cells transfected with LV-MST1 compared with the NC group (Fig. 4A and B). In addition, the number of cells arrested in the $\mathrm{G}_{1}$ phase was significantly increased, and the number of cells arrested in the $S$ phase was significantly decreased in LV-MST1-transfected cells compared with NC-transfected cells (Fig. 4C and D).

Inhibitor of the Hippo signaling pathway reverses MST1-induced effects in BCa. To determine whether the Hippo signaling pathway mediated the biological functions of MST1 in BCa, the Hippo signaling pathway inhibitor, XMU-MP-1 (an inhibitor of MST1/2), was used to inhibit the function of the Hippo signaling pathway in $\mathrm{BCa}$ cells overexpressing MST1. RT-qPCR analysis demonstrated that the treatment of MST1-overexpressing cells with the inhibitor downregulated the expression levels of MST1 (Fig. 5A). The results of the CCK- 8 and EdU incorporation assays revealed 

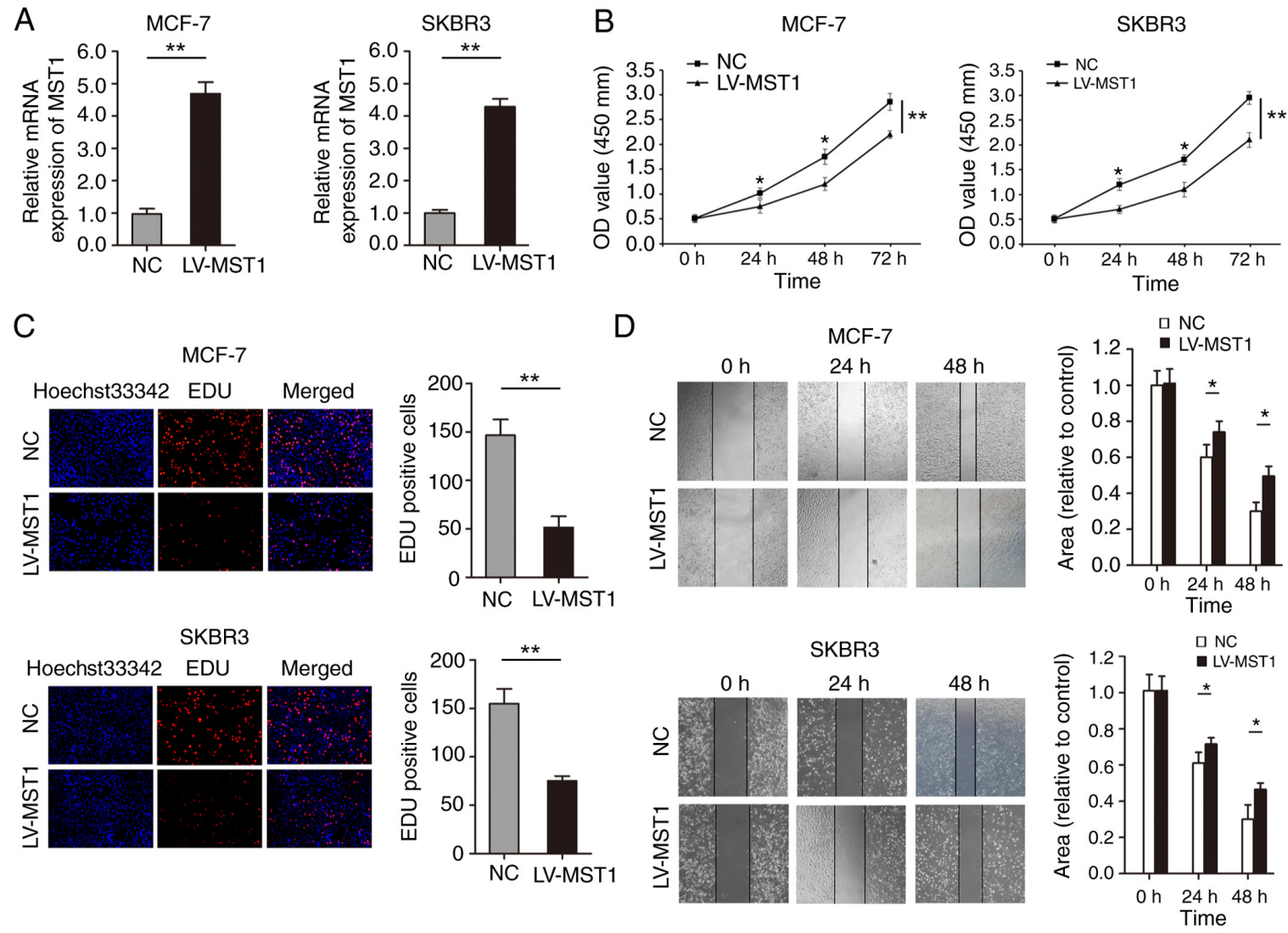

Figure 3. Overexpression of MST1 regulates the proliferation, migration and apoptosis of BCa cell lines. (A) Transfection efficiency of MCF-7 and SKBR3 cells transfected with LV-MST1 was determined using reverse transcription-quantitative PCR. (B) Cell Counting Kit-8 and (C) EdU incorporation assays (magnification, $\mathrm{x} 200$ ) were performed to determine the effects of MST1 overexpression on the proliferation of BCa cells. (D) Wound healing assays were conducted to determine the effects of MST1 overexpression on BCa cell migration (magnification, $\mathrm{x} 100$ ). ${ }^{*} \mathrm{P}<0.05,{ }^{* *} \mathrm{P}<0.01$. MST1, mammalian STE20-like protein kinase 1; $\mathrm{BCa}$, breast cancer; LV, lentivirus; OD, optical density; $\mathrm{NC}$, negative control; EdU, 5-Ethynyl-2'-deoxyuridine.

that the inhibited proliferative ability in the LV-MST1 group was partially restored in the LV-MST1 + XMU-MP-1 group (Fig. 5B and C). BCa cell migration was analyzed using wound healing assays; the results revealed that cell migration was also significantly increased in the MST1 + XMU-MP-1 cell group compared with the LV-MST1 group (Fig. 5D). Finally, western blotting analysis was performed to analyze the expression levels of key proteins in the Hippo signaling pathway. The expression levels of LATS1 and Bax were significantly downregulated, while the expression levels of YAP, Bcl-2 and Ki-67 were significantly upregulated in the LV-MST1 + XMU-MP-1 group compared with the LV-MST1 group in both cell lines (Fig. 6A and B).

\section{Discussion}

$\mathrm{BCa}$ is one of the most common types of malignant tumor in women, and its incidence continues to increase annually. In women, there were 2.26 million new cases of breast cancer worldwide in 2019, and BCa ranks second in incidence after uterine cancer, thus it poses a significant threat to the health of women, especially as the age of onset is steadily decreasing $(2,20)$. Currently, the primary prevention of $\mathrm{BCa}$ has not been achieved; therefore, strengthening secondary prevention, early detection, early diagnosis and early treatment methods may serve a vital role in reducing the mortality rates of patients with $\mathrm{BCa}$, thereby improving the survival rates (24). Despite recent significant advances in understanding the mechanisms of $\mathrm{BCa}$ development and the improvement of treatment strategies, $\mathrm{BCa}$ remains the second leading cause of cancer-related mortality in women, and death is almost always due to metastasis (25). The prognosis of patients with metastatic $\mathrm{BCa}$ is poor, with an average 5-year survival rate of only $27 \%$ (26). Although the expression of certain molecular markers in the different histological subtypes of BCa have strong prognostic value (such as HER2, Ras and K-Ras), the primary treatment of BCa still comprises chemotherapy or radiotherapy, and surgical resection for isolated lesions; however, the long-term effects of these treatments on survival is limited (27). Although chemotherapy, radiotherapy and surgical resection control the growth of primary tumors, they are not effective in preventing recurrence. Therefore, it remains a priority to identify the underlying molecular mechanism of $\mathrm{BCa}$ progression to help devise more precise, targeted treatments for $\mathrm{BCa}$ and to discover novel prognostic markers.

MST1, which belongs to the human serine/threonine kinase family, shares considerable homology with budding yeast kinase sps1 and ste 2 in its kinase domain (28). MST1 was reported to render cells highly sensitive to death receptor-mediated apoptosis by accelerating the activation of caspase-3, thus promoting apoptosis (29). Another study revealed that MST1 was a strong predictor of $\mathrm{BCa}$ prognosis (30). In pancreatic 
A

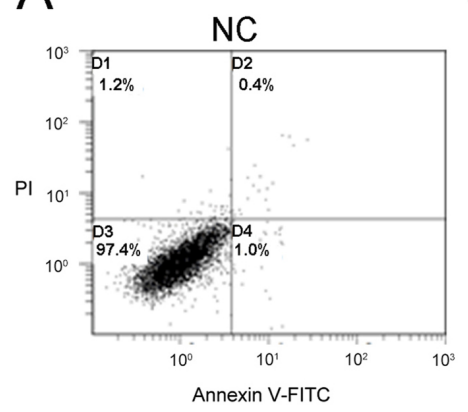

B

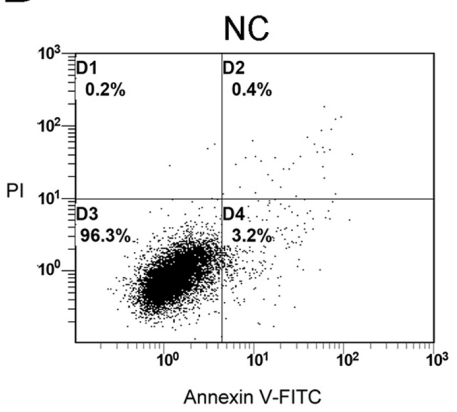

C

$\mathrm{NC}$

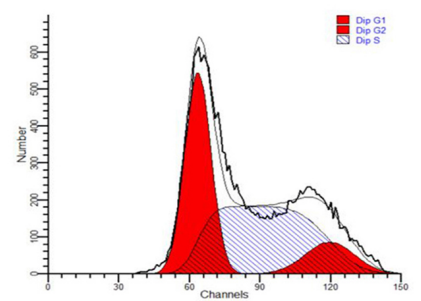

D

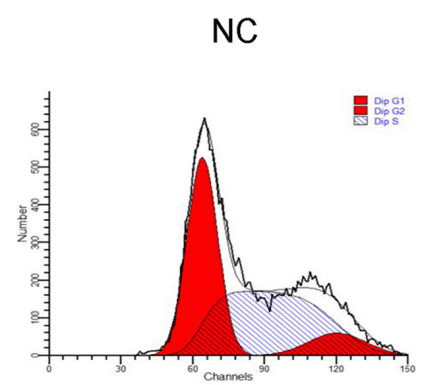

MCF-7
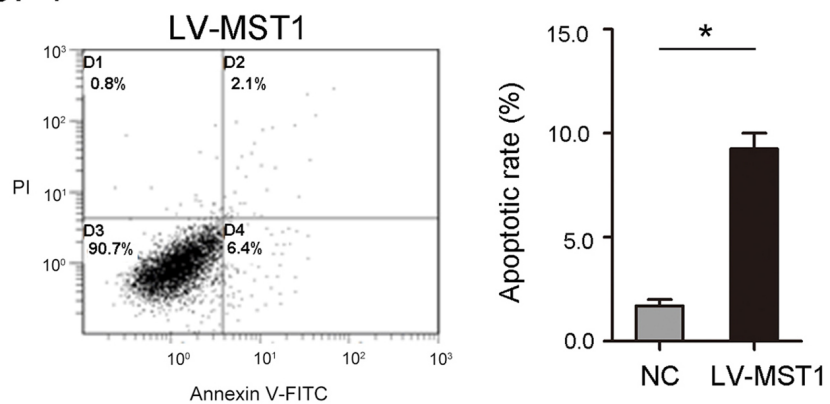

\section{SKBR3}
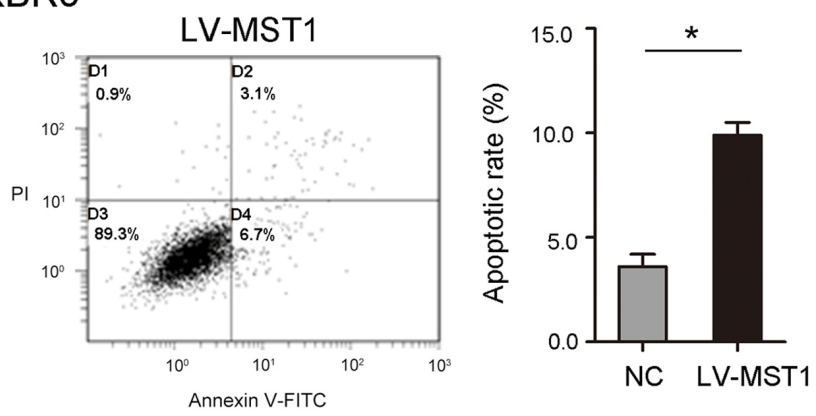

MCF-7

LV-MST1
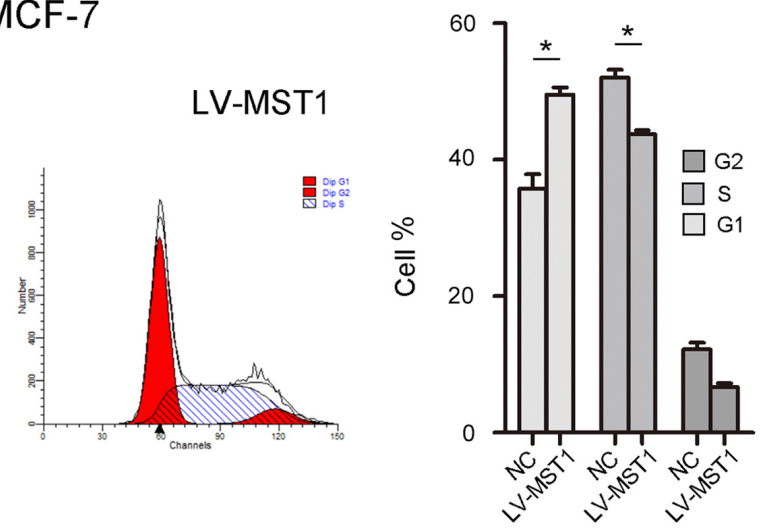

SKBR3

LV-MST1

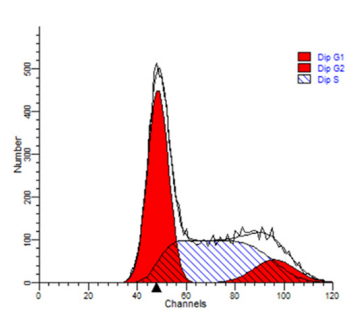

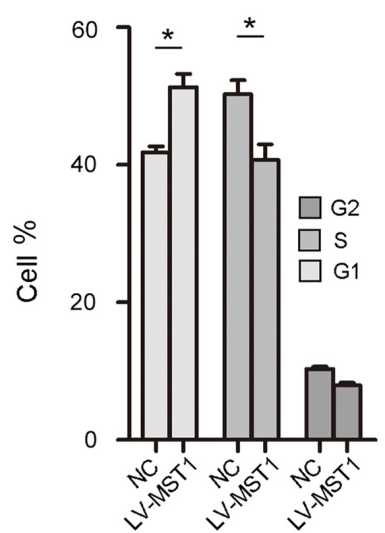

Figure 4. Overexpression of MST1 regulates cell cycle and apoptosis in breast cancer cell lines. Flow cytometry was used to determine the effects of MST1 overexpression on (A) MCF-7 and (B) SKBR3 cell apoptosis. Flow cytometry was used to analyze the cell cycle distribution of MST1-overexpressing (C) MCF-7 and (D) SKBR3 cells. "P<0.05. LV, lentivirus; MST1, mammalian STE20-like protein kinase 1; NC, negative control; PI, propidium iodide.

cancer, TNFreceptor-associated factor 6 promoted the degradation of MST1 through the ubiquitination degradation pathway, upregulated the expression levels of YAP and regulated the malignant proliferation and metastasis of tumor cells (31). In addition, the knockdown of microRNA (miR)-135b induced the apoptosis of gastric cancer cells by inhibiting the MAPK signaling pathway and upregulating the expression levels of MST1, thus inhibiting the proliferation of gastric cancer cells and increasing their resistance to cisplatin (32). Notably, in human HER2-positive BCa cells, the knockout of fibroblast 

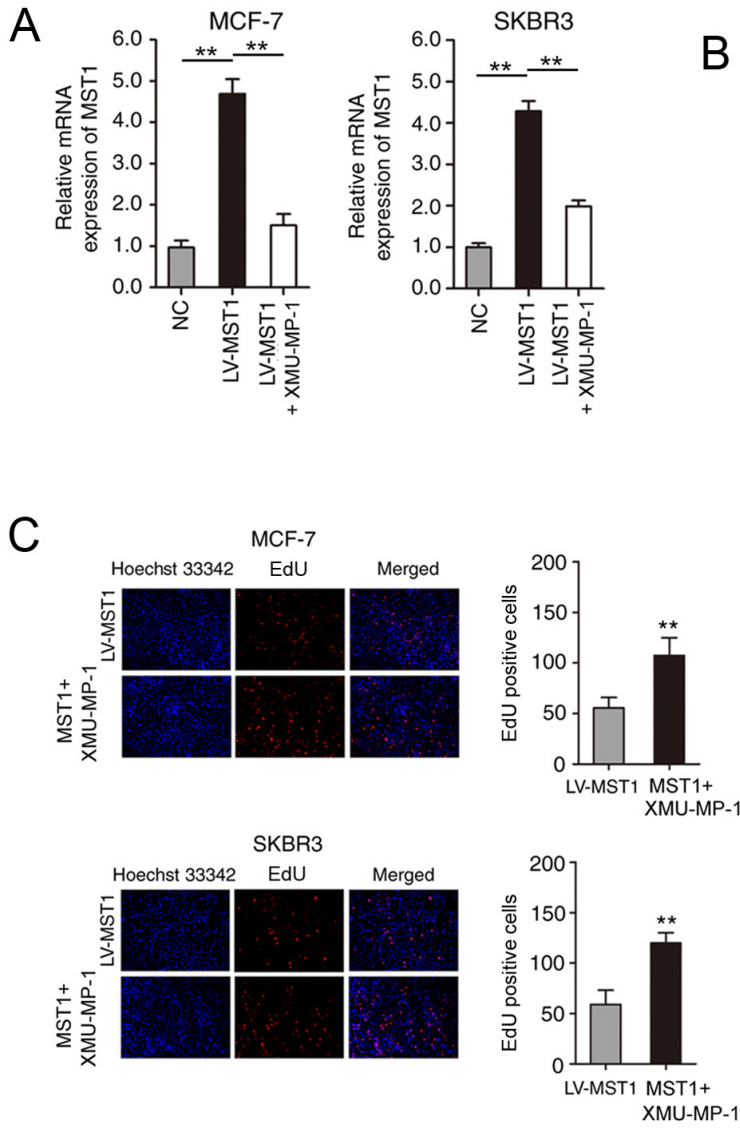

B

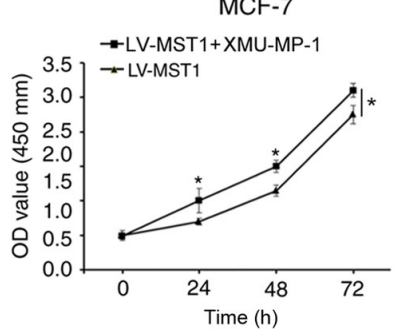

D
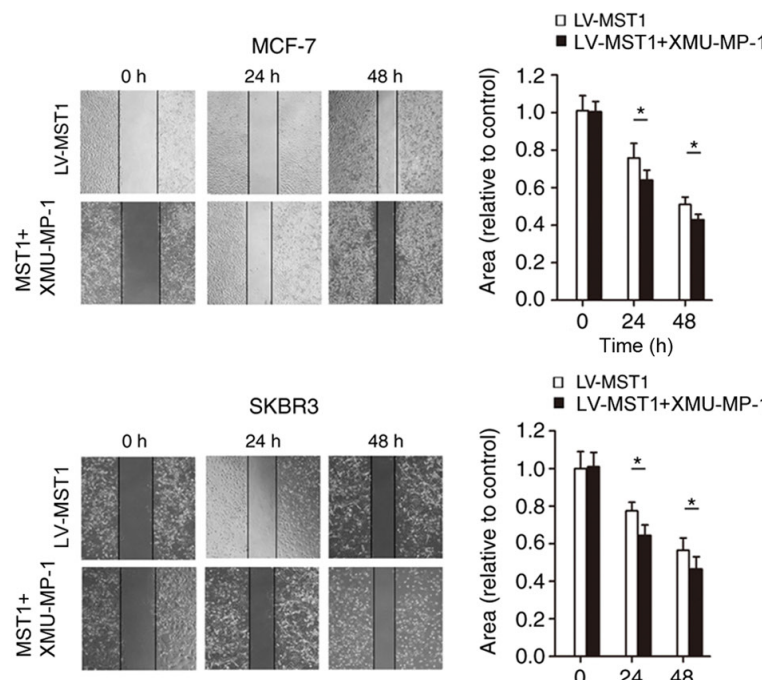

SKBR3

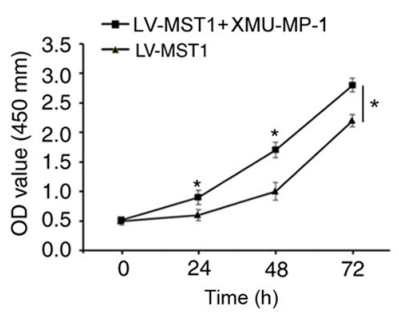

LV-MST1

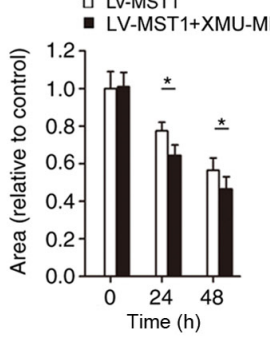

Figure 5. Hippo signaling pathway inhibitors reverse the effects of MST1 on breast cancer cells. (A) Reverse transcription-quantitative PCR was performed to analyze the expression levels of MST1 in the LV-MST1 and LV-MST1 + XMU-MP-1 groups of MCF-7 and SKBR3 cell lines. (B) Cell Counting Kit-8 assay was performed to detect the effect of LV-MST1 + XMU-MP-1 on the proliferation of MCF-7 and SKBR3 cell lines. (C) EdU incorporation assays were performed to determine the effects of LV-MST1 + XMU-MP-1 on the intake of EdU in MCF-7 and SKBR3 cell lines (magnification, x200). (D) Wound healing assay was performed to detect the effects of LV-MST1 + XMU-MP-1 on the migration of MCF-7 and SKBR3 cell lines (magnification, $\mathrm{x} 100)$. ${ }^{*} \mathrm{P}<0.05,{ }^{* *} \mathrm{P}<0.01$ (vs. LV-MST1 in part C). EdU, 5-Ethynyl-2'-deoxyuridine; LV, lentivirus; MST1, mammalian STE20-like protein kinase 1; NC, negative control; OD, optical density.

growth factor receptor 4 led to the activation of MST1 and increased the nuclear localization of MST1, resulting in N-terminal cleavage and autophosphorylation of MST1, which subsequently induced BCa cell apoptosis (33).

The results of the present study were similar to those reported previously. A large number of clinical BCa tissues were collected and the association between MST1 expression and the clinicopathological characteristics and the poor prognosis of patients with BCa were analyzed. In most cases, mRNA expression levels are strongly associated with the protein expression levels; however, due to gene post-transcriptional modifications or regulation, the balance between mRNA and protein expression levels can be disrupted. All the biological functions of MST1 depend on the expression of the protein (34); therefore, the present study analyzed the association between MST1 protein expression and prognosis using immunohistochemistry staining instead of determining mRNA expression levels. The results of the current study revealed that MST1 expression levels were significantly downregulated in $\mathrm{BCa}$ tissues and were significantly associated with the poor prognosis of patients. The expression levels of MST1 were also analyzed in BCa cell lines, in which MST1 was overexpressed using lentiviral vectors. MST1 overexpression significantly inhibited the proliferation and migration of $\mathrm{BCa}$ cells, and promoted apoptosis, indicating that MST1 may act as a tumor suppressor. The Hippo signaling pathway inhibits cell growth (35). In mammals, the upstream membrane protein receptor of the Hippo signaling pathway senses extracellular growth inhibition signals (36). Once the extracellular growth inhibition signal is sensed, a series of kinase cascade phosphorylation reactions are activated to result in the phosphorylation of the downstream effectors, YAP and TAZ. Subsequently, cytoskeletal proteins bind to phosphorylated YAP and TAZ, inducing their retention in the cytoplasm and a decrease in their nuclear activity to regulate organ size and cell growth (37). In hepatocellular carcinoma, miR-29c-3p was found to inhibit the invasion and metastasis of hepatocellular carcinoma cells through the Hippo signaling pathway (38). In addition, the interaction between WWC family member 3 (WWC3) and disheveled reduced the interaction between WWC3 and LATS1, which subsequently reduced the phosphorylation of LATS1, increased the nuclear import of YAP, and weakened the role of Hippo signaling pathway in lung cancer cells (39). MST1 is a key factor in the Hippo signaling pathway (39). XMU-MP-1 is an inhibitor of the sterile 20-like kinases MST1, which can inhibit MST1 activity, activate the downstream effector YAP and promote cell growth (40). In the present study, the XMU-MP-1 pathway inhibitor was used 

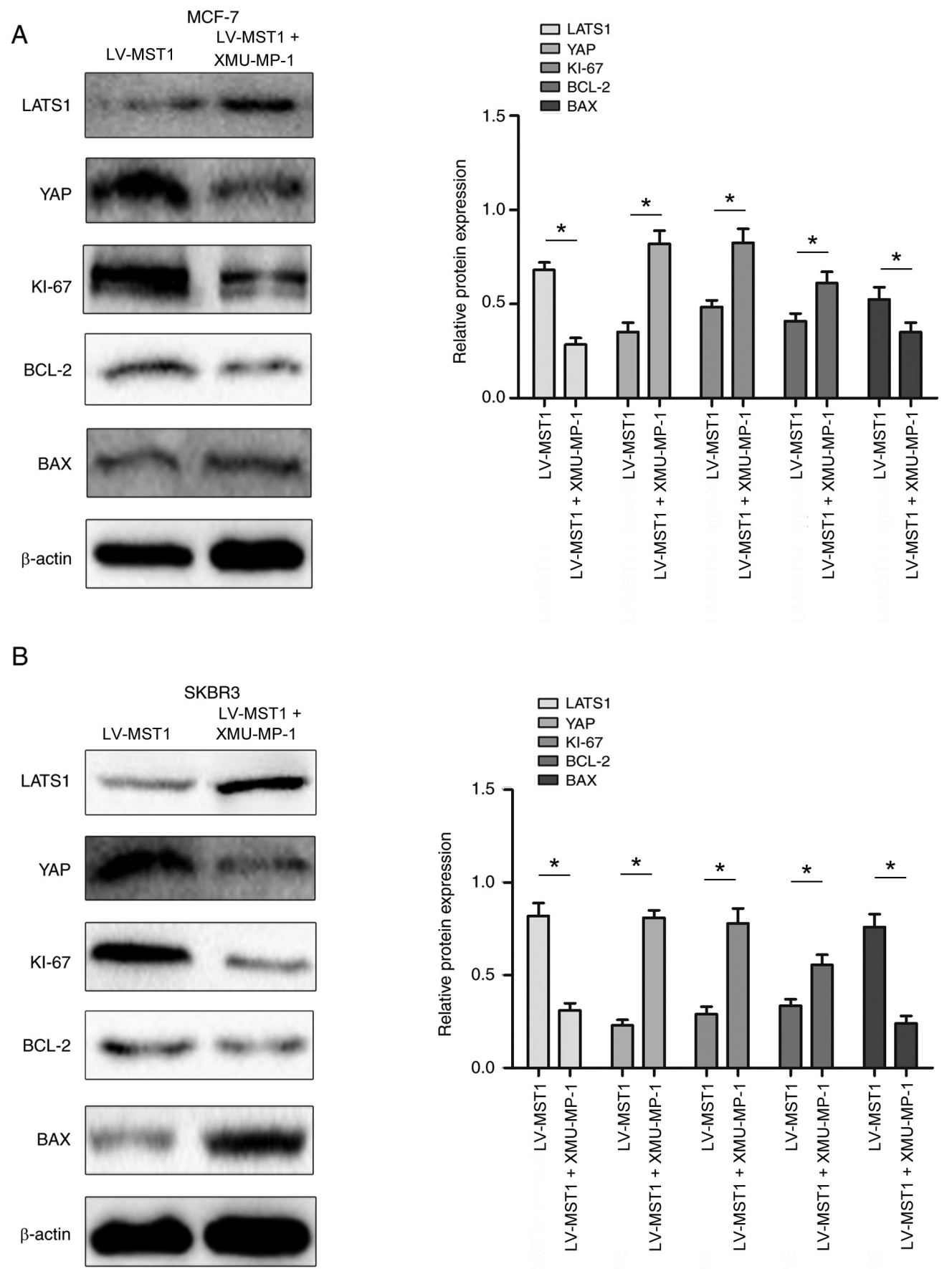

Figure 6. Potential mechanism through which MST1 was hypothesized to regulate the Hippo signaling pathway in breast cancer cells. Western blotting was used to analyze the protein expression levels of Ki-67, Bcl-2, Bax and intermediates of the Hippo signaling pathway, YAP and LATS1, in (A) MCF-7 and (B) SKBR3 cells following the transfection with LV-MST1 in the presence or absence of XMU-MP-1 treatment. "P<0.05. LATS1, large tumor suppressor kinase 1; LV, lentivirus; MST1, mammalian STE20-like protein kinase 1; YAP, YY1 associated protein 1.

to further verify whether MST1 inhibited the progression of BCa through the Hippo signaling pathway. XMU-MP-1 successfully reversed the inhibitory effect of MST1 on BCa cell functions (proliferation, migration and apoptosis). Subsequently, the expression levels of protein involved in the Hippo signaling pathway were altered, that is, the expression levels of LATS1 were downregulated, while the expression of YAP was upregulated, indicating that MST1 may play a role in $\mathrm{BCa}$ through the Hippo signaling pathway. However, there are some limitations to the study. For example, further investigations into how MST1 affected the Hippo signaling pathway and how it altered the expression levels of proliferation-related proteins and the apoptotic process (except for the expression levels of Bax and $\mathrm{Bcl}-2$ ) were not performed. Future studies will aim to determine other influencing factors that may affect the role of MST1 in BCa, which may provide further evidence that MST1 could be used as a prognostic biomarker in BCa.

In conclusion, the findings of the present study revealed that MST1 expression levels were significantly downregulated in BCa tissues and cells, and were closely associated with tumor size and clinical stage. The downregulated expression was also closely associated with the poor prognosis of patients with BCa. 
Mechanistically, the present findings suggested that MST1 may inhibit BCa cell proliferation and migration and promote its apoptosis by regulating the Hippo signaling pathway.

\section{Acknowledgements}

Not applicable.

\section{Funding}

The present study was supported by grants from The Key Research Projects on Application Foundation of Sichuan Science and Technology Department (grant no. 2017J Y0030) and Yibin Health and Family Planning Commission (grant no. 2017ZB031).

\section{Availability of data and materials}

The datasets used and/or analyzed during the current study are available from the corresponding author on reasonable request.

\section{Authors' contributions}

XJ and MX conceptualized and designed the study. XJ, LZ and SX made substantial contributions to the conception and design of the study, performed the experiments, and analyzed and interpreted the data. XJ and MX wrote the manuscript. ZC, JT and JY analyzed the data and performed the statistical analysis. All authors read and approved the manuscript, and take public responsibility for appropriate portions of the content. XJ and MX confirm the authenticity of all the raw data.

\section{Ethics approval and consent to participate}

The present study was approved by the Ethics Committee of The First People's Hospital of Yibin (Yibin, China; approval no. 20121011). Written informed consent was obtained from all patients prior to participation in the study.

\section{Patient consent for publication}

Not applicable.

\section{Competing interests}

The authors declare that they have no competing interests.

\section{References}

1. Harbeck N and Gnant M: Breast cancer. Lancet 18: 1134-1150, 2017.

2. Britt KL, Cuzick J and Phillips KA: Key steps for effective breast cancer prevention. Nat Rev Cancer 20: 417-436, 2020.

3. McGuire A, Brown JA, Malone C, McLaughlin R and Kerin MJ: Effects of age on the detection and management of breast cancer. Cancers (Basel) 7: 908-29, 2015.

4. Heer E, Harper A, Escandor N, Sung H, McCormack V and Fidler-Benaoudia MM: Global burden and trends in premenopausal and postmenopausal breast cancer: A population-based study. Lancet Glob Health 8: e1027-e1037, 2020.

5. Anastasiadi Z, Lianos GD, Ignatiadou E, Harissis HV and Mitsis M: Breast cancer in young Women: An overview. Updates Surg 69: 313-317, 2017.
6. Li Q, Liu J, Jiang Z and Liu Q: CSCO breast cancer guideline: Precise, economical and oriental. Sci China Life Sci 63: 1-3, 2020.

7. Winters S, Martin C, Murphy D and Shokar NK: Breast cancer epidemiology, prevention, and screening. Prog Mol Biol Transl Sci 151: 1-32, 2017.

8. Galan JA and Avruch J: MST1/MST2 Protein Kinases: Regulation and physiologic roles. Biochemistry 55: 5507-5519, 2016.

9. Hui OY, Zhou EX and Wang H: Mst1-Hippo pathway triggers breast cancer apoptosis via inducing mitochondrial fragmentation in a manner dependent on JNK-Drp1 axis. Onco Targets Ther 12: 1147-1159, 2019.

10. Harvey KF, Zhang X and Thomas DM: The Hippo pathway and human cancer. Nat Rev Cancer 13: 246-257, 2013.

11. Hong AW, Meng Z and Guan KL: The Hippo pathway in intestinal regeneration and disease. Nat Rev Gastroenterol Hepatol 13: 324-337, 2016.

12. Torres-Bacete J, Delgado-Martin C, Gomez-Moreira C, Simizu S and Rodríguez-Fernández JL: The mammalian sterile 20-like 1 kinase controls selective CCR7-dependent functions in human dendritic cells. J Immunol 195: 973-81, 2015.

13. Zhang M, Zhang L, Hu J, Lin J, Wang T, Duan Y, Man W, Feng J, Sun L, Jia H, et al: MST1 coordinately regulates autophagy and apoptosis in diabetic cardiomyopathy in mice. Diabetologia 59: 2435-2447, 2016.

14. Zhao WB, Lu Q, Nguyen MN, Su Y, Ziemann M, Wang LN, Kiriazis H, Puthalakath H, Sadoshima J, Hu HY and Du XJ: Stimulation of $\beta$-adrenoceptors up-regulates cardiac expression of galectin-3 and BIM through the hippo signalling pathway. $\mathrm{Br}$ J Pharmacol 176: 2465-2481, 2019.

15. Singh A, Ramesh S, Cibi DM, Yun LS, Li J, Li L, Manderfield LJ, Olson EN, Epstein JA and Singh MK: Hippo signaling mediators yap and taz are required in the epicardium for coronary vasc $\mu 1$ ature development. Cell Rep 15: 1384-1393, 2016.

16. Yu FX, Zhao B and Guan KL: Hippo pathway in organ size control, tissue homeostasis, and cancer. Cell 163: 811-828, 2015.

17. Nallet-Staub F, Marsaud V, Li L, Gilbert C, Dodier S, Bataille V, Sudol M, Herlyn M and Mauviel A: Pro-invasive activity of the Hippo pathway effectors YAP and TAZ in cutaneous melanoma. J Invest Dermatol 134: 123-132, 2014.

18. He M, Zhou Z, Shah AA, Hong Y, Chen Q and Wan Y: New insights into posttranslational modifications of Hippo pathway in carcinogenesis and therapeutics. Cell Div 11: 4, 2016.

19. Vincent-Mistiaen Z, Elbediwy A, Vanyai H, Cotton J, Stamp G, Nye E, Spencer-Dene B, Thomas GJ, Mao J and Thompson B: YAP drives cutaneous squamous cell carcinoma formation and progression. Elife 7: e33304, 2018.

20. Maugeri-Saccà M and De Maria R: Hippo pathway and breast cancer stem cells. Crit Rev Oncol Hematol 99: 115-122, 2016.

21. Rodriguez D, Ramkairsingh M, Lin X, Kapoor A, Major P and Tang D: The central contributions of breast cancer stem cells in developing resistance to endocrine therapy in estrogen receptor (ER)-positive breast cancer. Cancers (Basel) 11: 1028, 2019.

22. Cortes E, Lachowski D, Rice A, Thorpe SD, Robinson B, Yeldag G, Lee DA, Ghemtio L, Rombouts K and Del Río Hernández AE: Tamoxifen mechanically deactivates hepatic stellate cells via the $G$ protein-coupled estrogen receptor. Oncogene 38: 2910-2922, 2019.

23. Livak KJ and Schmittgen TD: Analysis of relative gene expression data using real-time quantitative PCR and the 2(-Delta Delta C(T)) method. Methods 25: 402-408, 2001.

24. Kolak A, Kamińska M, Sygit K, Budny A, Surdyka D, Kukiełka-Budny B and Burdan F: Primary and secondary prevention of breast cancer. Ann Agric Environ Med 24: 549-553, 2017.

25. Hosonaga M, Saya H and Arima Y: Molecular and cellular mechanisms underlying brain metastasis of breast cancer. Cancer Metastasis Rev 39: 711-720, 2020.

26. Siegel RL, Miller KD and Jemal A: Cancer statistics. 2016. CA Cancer J Clin 66: 7-30, 2016.

27. McDonald ES, Clark AS, Tchou J, Zhang P and Freedman GM: Clinical diagnosis and management of breast cancer. J Nucl Med 57 (Suppl 1): 9S-16S, 2016.

28. Kim MK, Jang JW and Bae SC: DNA binding partners of YAP/TAZ. BMB Rep 51: 126-133, 2018.

29. He Z, Zhao TT, Jin F, Li JG, Xu YY, Dong HT, Liu Q, Xing $\mathrm{P}, \mathrm{Zhu} \mathrm{GL}, \mathrm{Xu} \mathrm{H}$ and Miao ZF: Downregulation of RASSF6 promotes breast cancer growth and chemoresistance through regulation of Hippo signaling. Biochem Biophys Res Commun 503: 2340-2347, 2018 . 
30. Li JA, Kuang T, Pu N, Fang Y, Han X, Zhang L, Xu X, Wu W, Wang D, Lou W and Rong Y: TRAF6 Regulates YAP signaling by promoting the ubiquitination and degradation of MST1 in pancreatic cancer. Clin Exp Med 19: 211-218, 2019.

31. Lin XY, Cai FF, Wang MH, Pan X, Wang F, Cai L, Cui RR, Chen S and Biskup E: Mammalian sterile 20-like kinase 1 expression and its prognostic significance in patients with breast cancer. Oncol Lett 14: 5457-5463, 2017.

32. Zhou J and Che Q: Poor expression of microRNA-135b results in the inhibition of cisplatin resistance and proliferation and induces the apoptosis of gastric cancer cells through MST1-mediated MAPK signaling pathway. FASEB J 33: 3420-3436, 2019.

33. Turunen SP, Nandelstadh PV, Öhman T, Gucciardo E, Seashore-Ludlow B, Martins B, Rantanen V, Li H, Höpfner K, Östling P, et al: FGFR4 phosphorylates MST1 to confer breast cancer cells resistance to MST1/2-dependent apoptosis. Cell Death Differ 26: 2577-2593, 2019.

34. Buccitelli $\mathrm{C}$ and Selbach M: mRNAs, proteins and the emerging principles of gene expression control. Nat Rev Genet 21: 630-644, 2020.

35. Kulkarni A, Chang MT, Vissers JHA, Dey A and Harvey KF: The hippo pathway as a driver of select human cancers. Trends Cancer 6: 781-796, 2020.
36. Zheng Y and Pan D: The hippo signaling pathway in development and disease. Dev Cell 50: 264-282, 2019.

37. Tao Y, Cai F, Shan L, Jiang H, Ma L and Yu Y: The hippo signaling pathway: An emerging anti-cancer drug target. Discov Med 24: 7-18, 2017.

38. Wu H, Zhang W, Wu Z, Liu Y, Shi Y, Gong J, Shen W and Liu C: miR-29c-3p regulates DNMT3B and LATS1 methylation to inhibit tumor progression in hepatocellular carcinoma. Cell Death Dis 10: 48, 2019.

39. Han Q, Lin X, Zhang X, Jiang G, Zhang Y, Miao Y, Rong X, Zheng $X$, Han Y, Han X, et al: WWC3 regulates the Wnt and hippo pathways via dishevelled proteins and large tumour suppressor 1, to suppress lung cancer invasion and metastasis. J Pathol 242: 435-447, 2017.

40. Fan F, He Z, Kong LL, Chen Q, Yuan Q, Zhang S, Ye J, Liu H, Sun X, Geng J, et al: Pharmacological targeting of kinases MST1 and MST2 augments tissue repair and regeneration. Sci Transl Med 8: 352ra108, 2016.

(i) (9) This work is licensed under a Creative Commons

Attribution-NonCommercial-NoDerivatives 4.0 International (CC BY-NC-ND 4.0) License. 